\title{
Caveolin-1 plays a key role in the oleanolic acid-induced apoptosis of HL-60 cells
}

\author{
WEI MA ${ }^{1,2^{*}}$, DI-DI WANG ${ }^{1}$, LI LI ${ }^{1}$, YU-KUAN FENG ${ }^{2}$, HONG-MEI GU ${ }^{2}$, GUI-MING ZHU ${ }^{1}$, \\ JIN-HUA PIAO ${ }^{1}$, YU YANG $^{1}, \mathrm{XU} \mathrm{GAO}^{3}$ and PENG-XIA ZHANG ${ }^{1 *}$ \\ ${ }^{1}$ School of Basic Medicine, Jiamusi University, Jiamusi $154000 ;{ }^{2}$ Department of Biology, \\ Mudanjiang Medical University, Mudanjiang 157011; ${ }^{3}$ Department of Biochemistry, \\ Harbin Medical University, Harbin 150086, P.R. China
}

Received March 10, 2014; Accepted April 14, 2014

DOI: $10.3892 /$ or.2014.3177

\begin{abstract}
Our previous study found that caveolin-1 (CAV-1) protein expression is upregulated during oleanolic acid (OA)-induced inhibition of proliferation and promotion of apoptosis in HL-60 cells. CAV-1 is the main structural protein component of caveolae, playing important roles in tumorigenesis and tumor development. It has been shown that cav-1 expression is lower in leukemia cancer cell lines SUP-B15, HL-60, THP-1 and K562 and in chronic lymphocytic leukemia primary (CLP) cells when compared with normal white blood cells, with the lowest cav-1 expression level found in HL-60 cells. To study the effects of cav-1 in HL-60 cells and the effects of cav-1 overexpression on OA drug efficacy, cav-1 was overexpressed in HL-60 cells using lentiviral-mediated transfection combined with OA treatment. The results showed that cav-1 overexpression inhibited HL-60 cell proliferation, promoted apoptosis, arrested the cell cycle in the G1 phase and inhibited activation of the PI3K/AKT/mTOR signaling pathway. Overexpression of CAV-1 also increased HL-60 cell sensitivity to OA. To further verify whether OA affects HL-60 cells via the activation of downstream signaling pathways by CAV-1, cav-1 gene expression was silenced using RNAi, and the cells were treated with OA to examine its efficacy. The results showed that after cav-1 silencing, OA had little effect on cell activity, apoptosis, the cell cycle and phosphorylation of HL-60 cells. This study is the first to show that CAV-1 plays a crucial role in the effects of OA on HL-60 cells.
\end{abstract}

Correspondence to: Professor Peng-Xia Zhang, School of Basic Medicine, Jiamusi University, Jiamusi, Heilongjiang 154000, P.R. China

E-mail: pengxiaz@163.com

*Contributed equally

Key words: CAV-1, overexpression, RNA interference, HL-60, AML, oleanolic acid, lentivirus, PI3K

\section{Introduction}

Acute myelogenous leukemia (AML) is a malignant neoplastic disease caused by the abnormal proliferation of hematopoietic stem cells. Although treatments for AML have made considerable progress in recent years, more than $20 \%$ of patients still cannot be completely cured; they eventually relapse and succumb to the disease. It is known that cancerous cells undergo indefinite cell proliferation caused by disorders of signal transduction pathways (1). The success of imatinib and other drugs in the treatment of chronic myelogenous leukemia has demonstrated that targeted therapy of leukemia is an important therapeutic development.

Oleanolic acid (OA) is a pentacyclic triterpenoid; the molecular formula is $\mathrm{C}_{30} \mathrm{H}_{48} \mathrm{O}_{3}$. OA was first separated from the leaves of a clover plant, Olea europeae L, in 1908. Since the identification of OA as an effective anti-hepatitis monomer, new biological properties have been discovered, including blood sugar and blood lipid lowering abilities, as well as anti-bacterial, anti-viral, anti-caries (2), immunoregulatory (3), anti-oxidative, anti-mutation, anti-cardiovascular and cerebrovascular disease (4), scar formation inhibitory (5) and antitumor effects. In recent years, the antitumor effects of OA have attracted increased attention. OA shows an inhibitory effect on solid tumors including skin cancer, lung cancer, breast cancer and osteosarcomas. The mechanisms of the antitumor effects of OA are complicated; it is involved in the regulation of a variety of signal transduction pathways. ERK-P53 (6), mTOR (7), Bcl-2 (8,9), VEGF (10), NO and TNF- $\alpha$ (11) can all participate in the anticancer activity of OA. Our previous study found that inhibition of proliferation and the promotion of apoptosis in OA-treated HL-60 cells were accompanied by increased caveolin-1 (CAV-1) expression and inhibition of the $\mathrm{PI} 3 \mathrm{~K} / \mathrm{AKT}$ signal transduction pathway.

The human cav-1 gene is located on chromosome 7q31.1. The molecular weight of CAV-1 is $22 \mathrm{kDa}$. CAV-1 binds to a variety of signaling proteins and inhibits their activities; therefore, it is considered a 'broad spectrum' kinase inhibitor located at the center of various signaling pathways (12). Cav-1 is expressed in a variety of tumor cells with significantly different expression patterns and dual carcinogenic and tumorsuppressor functions. CAV-1 has a variety of biological effects, 
Table I. Primer sequences and annealing temperatures for quantitative RT-PCR.

\begin{tabular}{|c|c|c|c|c|}
\hline Gene name (accession no.) & Primer name & Primer sequence (5'-3') & Temperature $\left({ }^{\circ} \mathrm{C}\right)$ & Product size (bp) \\
\hline \multirow[t]{2}{*}{$\beta$-actin (NM_001101) } & Forward primer & CCTGTACGCCAACACAGTGC & \multirow[t]{2}{*}{60} & \multirow[t]{2}{*}{211} \\
\hline & Reverse primer & ATACTCCTGCTTGCTGATCC & & \\
\hline \multirow[t]{2}{*}{ cav-1 (NM_001753.4) } & Forward primer & CGGGAACAGGGCAACATGTACA & \multirow[t]{2}{*}{58} & \multirow[t]{2}{*}{178} \\
\hline & Reverse primer & TCССТTCTGGTTCTGCAATCACAT & & \\
\hline
\end{tabular}

including affecting tumor multidrug resistance $(13,14)$, regulating the cell cycle (15) and signal transduction, participating in the regulation of aging $(16,17)$, enhancing cell autophagy function $(18,19)$ and participating in cell proliferation, apoptosis, migration and differentiation processes. In leukemia patients, CAV-1 and MDR-1 expression levels are positively correlated (20), and external stimulating factors such as HTLV can induce CAV-1 overexpression in T-cell lines (21). In untreated AML patients, $\mathrm{CAV}-1$ expression levels were found to be significantly lower than levels in the normal control group; the levels increased after remission from induction chemotherapy and decreased again during relapse. Thus, cav-1 has high research value for the treatment of leukemia.

The goal of this study was to investigate the roles of cav-1 in HL-60 cells and to explore the mechanism of OA effects. The results showed that OA not only promoted CAV-1 expression in HL-60 cells but also increased protein phosphorylation. To further study whether OA affects HL-60 cells by activating downstream signaling pathways via CAV-1, the lentiviral overexpression vector pcDNA-CAV-1 and the shRNA expression vector PSIH1-H1-CAV-1 were constructed to overexpress and silence cav-1 in HL-60 cells, respectively. The cav-1-mediated cell proliferation and apoptosis and the PI3K/AKT pathway were examined; OA efficacy was also determined in HL-60 cells overexpressing cav- 1 . At same time, the role of OA after cav-1 silencing was determined. The results showed that OA, cav-1 overexpression or their combination in HL-60 cells inhibited proliferation, promoted apoptosis, arrested the cell cycle in the G1 phase, downregulated P110 $\alpha$, p-AKT (Thr308), p-AKT (Ser473) and mTOR and upregulated p-CAV-1 levels. The effects of the joint treatment were more significant. After cav-1 silencing, OA showed no effect on HL-60 cell proliferation, apoptosis, cell cycle and levels of P110 $\alpha$, p-AKT (Thr308), p-AKT (Ser473), mTOR and p-CAV-1.

\section{Materials and methods}

Cell culture and detection of cav-1 expression in various cell lines. The human leukemia cell lines SUP-B15, HL-60, THP-1 and K562 were purchased from the Chinese Academy of Sciences Cell Library. The cells were cultured in RPMI-1640 culture medium containing $10 \%$ fetal bovine serum at $37^{\circ} \mathrm{C}$ and $5 \% \mathrm{CO}_{2}$. The cells were passaged every 2-3 days. The cells were collected at the logarithmic growth phase. CLL primary tumor cells (CLP) and white blood cells (WBCs) were collected through centrifugation directly after separation. Expression of cav-1 in each cell line was detected using qRT-PCR and western blot assays. Primers are listed in Table I.
Construction of recombinant lentiviral expression vector pcDNA-CAV-1 and pSIHI-HI-CAV-1. First, total-RNA was extracted from freshly prepped human leukocytes, and cav-1 cDNA with $\mathrm{Xba \textrm {I }}$ and BamHI restriction sites at both ends was amplified using PCR. The digested fragment was then inserted into pcDNA-EF1-GFP between the corresponding restriction sites to construct the recombinant plasmid pcDNA-CAV-1.

Second, three siRNA sequences targeting the cav-1 (NM_001753.4) CDS region were designed and synthesized by Shanghai Invitrogen. The synthetic sequences were inserted into pSIH1-H1-GFP shRNA vectors to construct the recombinant pSIH1-CAV-1-shRNA plasmids (pSIH1-CAV-1shRNA1, pSIH1-CAV-1-shRNA2, pSIH1-CAV-1-shRNA3, pSIH1-CAV-1-Negative).

The positive clones of the above recombinant plasmids pcDNA-CAV-1 and pSIH1-CAV-1-shRNA were screened and sequenced, and the correct recombinant expression vector plasmid DNA was purified to eliminate the endotoxin. The selected 293 cells were transfected and divided into 7 groups: the blank control (293), the pcDNA empty vectortransfected cells (293-P), the pcDNA-CAV-1-transfected cells (293-PC) and four pcDNA-CAV-1 of pSIH1-CAV-1-shRNAtransfected cells (293-PC-S1, 293-PC-S2, 293-PC-S3, 293-PC-N). Lipofectamine 2000 was used as the transfection reagent. qRT-PCR and western blot assays were used to detect cav-1 expression levels and to screen effective siRNA sequences.

Recombinant lentiviral infection of HL-60 cells. The third generation of the lentiviral vector was used for viral packaging and production following the manufacturer's instructions. Briefly, 293TN cells were transfected with a mixture of lentiviral vector (pcDNA-CAV-1 or pSIH1-CAV-1-shRNA) and packaging plasmid, and the supernatant was collected and filtered using a $0.45 \mu \mathrm{mol} / 1 \mathrm{PVDF}$ membrane. Viral titers were determined through gradient centrifugation. After the high-titer viral preparation met the requirements, the host HL-60 cells were infected at an optimal MOI. CAV-1 expression levels in the infected HL-60 cells were detected through western blot assays. The GFP reporter gene was present in both vectors. Transfection efficiency was easily determined, as green fluorescence was a necessary prerequisite for a high efficiency infection of HL-60 cells.

OA treatment and grouping. HL-60 cell suspensions in the logarithmic growth phase before and after transfection were prepared and dispensed in 96-well plates, with a $100 \mu 1$ cell suspension containing $1 \times 10^{4}$ cells per well. The HL-60 cells 
$\mathbf{A}$

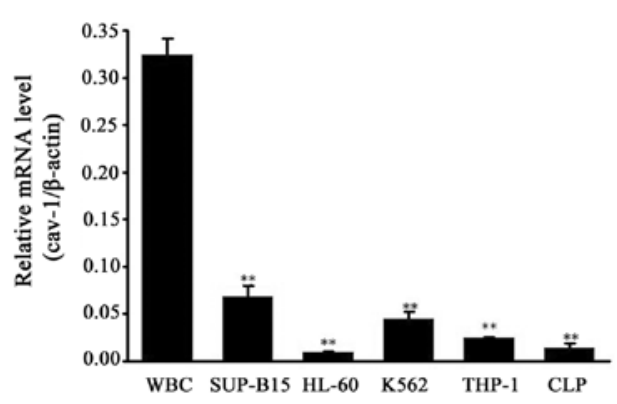

C

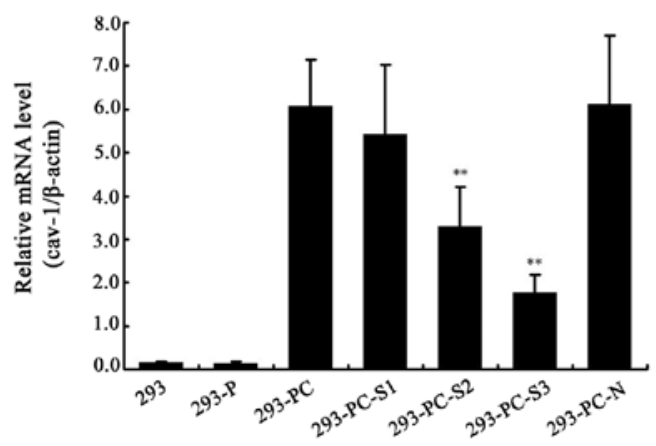

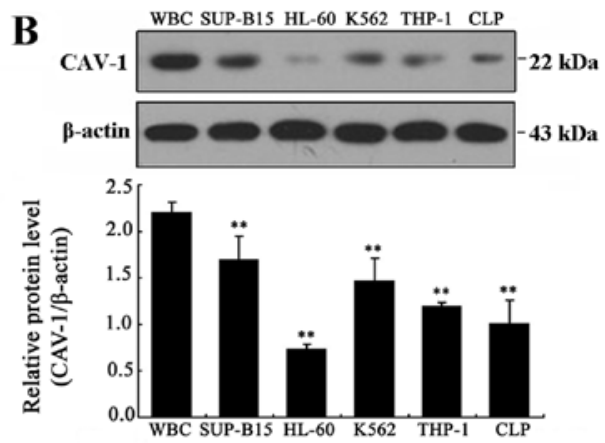

D
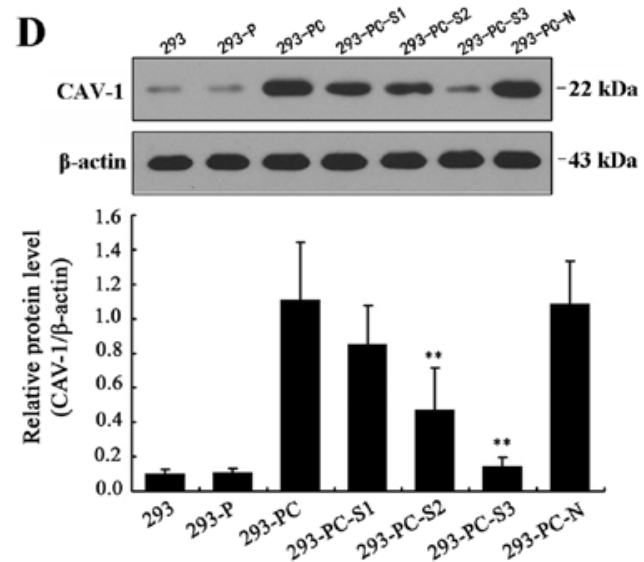

Figure 1. cav-1 expression in the leukemia tumor cell lines and reconstruction of the lentiviral expression vector. (A) cav-1 expression levels were detected by qRT-PCR. (B) CAV-1 expression levels were detected by Western blot assays. (C) cav-1 expression levels in 293 cells were detected by qRT-PCR. (D) CAV-1 expression levels in 293 cells were detected by western blot assays. The data indicate the mean \pm SD of triplicates; ${ }^{* *} \mathrm{P}<0.01$, samples compared with the control group (WBCs).

were divided into 10 groups: the control group (HL-60), the OA-treated HL-60 group (HL-60+OA), the empty pcDNA-transfected cell group (HL-60-L), the cav-1-overexpressing cell group (HL-60-LC), the OA-treated HL-60-L group (HL-60-L+OA), the OA-treated HL-60-LC group (HL-60-LC+OA), the pSIH1-CAV-1-shRNA3-transfected group (HL-60-LS3), the pSIH1-CAV-1-negative-transfected cell group (HL-60-LN), the OA-treated HL-60-LN group (HL-60-LN+OA) and the OA-treated HL-60-LS3 group (HL-60-LS3+OA). The final OA concentration for cell treatment was $80 \mu \mathrm{M} / 1$.

Western blot analyses. The cells were collected and lysed for protein extraction. Protein concentrations were determined using the BCA technique, and the cell lysates were subjected to SDS-PAGE electrophoresis. After separation, the proteins were transferred to PVDF membranes; the membranes were blocked using TBST containing 5\% non-fat dry milk. Then, the anti-human antibodies anti-CAV-1, P110 $\alpha$, p-AKT (Thr308), p-AKT (Ser473), p-CAV-1 (Tyr14) and mTOR were added. Horseradish peroxidase-conjugated anti-rabbit (or mouse) IgG (Cell Signaling Technology) was used for detection of immunoreactive proteins by chemiluminescence (Pierce ECL western blotting substrate).

Cell proliferation activity analysis. Dispensed cells of each group were placed into 96 -well plates with $1 \times 10^{4}$ cells per well; $10 \mu 1$ of CCK-8 reagent was added per well at 24,48 and
$72 \mathrm{~h}$. After culturing the treated plates in an incubator for $4 \mathrm{~h}$, the supernatants were transferred to another 96 -well plate, and the absorbance at $450 \mathrm{~nm}$ was measured using a microplate reader.

Flow cytometric analysis. The cells of each group were plated in 12-well plates, with $\sim 1 \times 10^{5}$ cells per well. The cells were incubated to $75-80 \%$ confluency per well. Cell apoptosis was detected using a double staining Annexin V:FITC Apoptosis Detection Kit II. The cells were harvested, washed with ice-cold PBS twice and fixed with $75 \%$ cold ethanol at $4{ }^{\circ} \mathrm{C}$ overnight. Propidium iodide (PI) staining of nuclei was used to monitor the phases of the cell cycle. The fluorescence of DNA-bound PI in cells was measured with a FACSCalibur flow cytometer (BD Biosciences).

Statistical analyses. The data are expressed as the mean \pm standard deviation (SD) and were compared by one-way analysis of variance (one-way ANOVA), Dunnet t-test and least significant difference-test (LSD). Statistical analyses were performed via SPSS statistical software $16.0 ; \mathrm{P}<0.05$ or $\mathrm{P}<0.01$ was considered to indicate a statistically significant difference.

\section{Results}

Cav-1 gene expression levels in various leukemia cell lines. The qRT-PCR and western blotting results revealed the cav-1 mRNA and protein expression levels in all tested cell lines: 

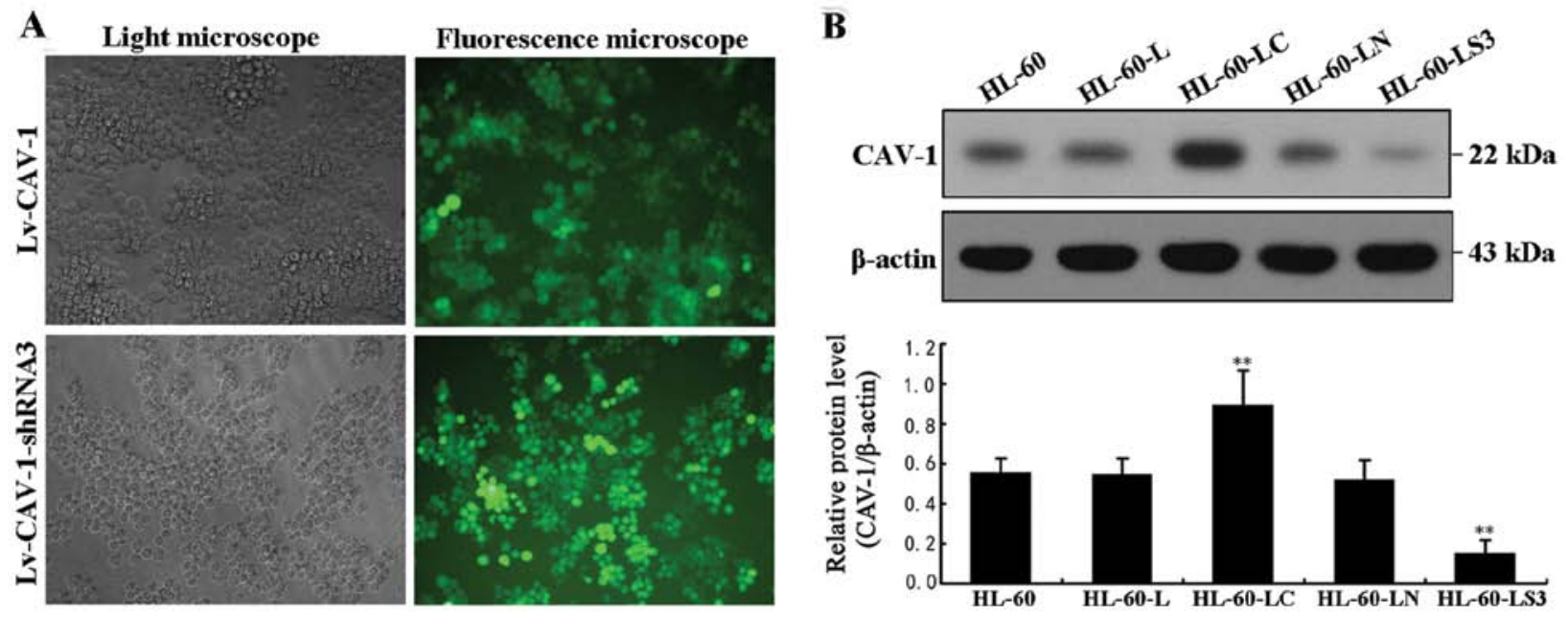

Figure 2. HL-60 cells were successfully transfected by Lv-CAV-1 and Lv-CAV-1-shRNA3. (A) Stably transfected HL-60 cells were easily identified by fluorescence microscopy after $72 \mathrm{~h} ; \mathrm{MOI}=20,200 \mathrm{x}$. (B) CAV-1 expression levels in HL-60 cells were detected by western blot assays. The data indicate the mean $\pm \mathrm{SD}$ of triplicates; ${ }^{* *} \mathrm{P}<0.01$, samples compared with the control group (HL-60).

WBC, SUP-B15, HL-60, K562, THP-1 and CLP. Compared with WBCs, cav-1 expression levels were reduced in the other cell lines, with the lowest cav-1 expression found in HL-60 cells (Fig. 1A and B). Thus, we chose the HL-60 cell line for subsequent experiments.

Construction of the recombinant vectors pcDNA-CAV-1 and pSIH1-H1-shRNA-CAV-1. The positive pcDNA-EF1-GFP clones containing cav-1 and the PSIH1-shRNA plasmid containing cav-1 siRNA were screened and sequenced. The results showed that the sequences were identical to that of the recombinant plasmid. The two vectors were successfully transfected into 293 cells using Lipo $2000^{\mathrm{TM}}$ and were effectively expressed. qRT-PCR results showed that the mRNA expression levels were significantly higher in the 293-PC cells than in the control cells. The three designed siRNA sequences were screened on the basis of cav-1 overexpression in the stably transfected 293 cell line. The screening results indicated that the 293-PC-S3 cells showed the strongest inhibition of cav-1 mRNA and protein expression levels among the tested cells (Fig. 1C and D). Therefore, we chose siRNA-3, which showed the highest cav-1 gene silencing efficiency, for subsequent experiments.

Lv-CAV-1 and Lv-CAV-1-shRNA3 successfully infected $H L-60$ cells. HL-60 cells were observed using green fluorescence microscopy $72 \mathrm{~h}$ after transfection. The transfection efficiencies of Lv-CAV-1 and Lv-CAV-1-shRNA3 were 85 and $90 \%$, respectively (Fig. 2A). Western blotting results $96 \mathrm{~h}$ after transfection (Fig. 2B) showed that CAV-1 protein levels were significantly increased in the HL-60-LC cells and significantly reduced in the HL-60-LS3 cells.

Cell proliferation. HL-60 cell activity was significantly decreased $72 \mathrm{~h}$ after the addition of OA, with absorbance values of $2.10 \pm 0.22$ vs. $1.48 \pm 0.12(\mathrm{P}<0.01)$. The activities of the HL-60-LC cells were decreased at 48 and $72 \mathrm{~h}$, with absorbance values of $1.32 \pm 0.12$ vs. $0.98 \pm 0.14(\mathrm{P}<0.05)$ and $2.10 \pm 0.22$ vs. $1.55 \pm 0.09(\mathrm{P}<0.01)$, respectively. The prolif- eration of HL-60-LC+OA cells decreased in the first $24 \mathrm{~h}$, with absorbance value of $0.49 \pm 0.10$ vs. $0.33 \pm 0.02(\mathrm{P}<0.05)$. Cell proliferation inhibition was more significant in the HL-60-LC+OA cells than in the HL-60+OA and HL-60-LC cells. These results indicated that both CAV-1 and OA inhibited HL-60 cell proliferation, and the inhibitory effect was more significant in the HL-60-LC+OA cells (Fig. 3A).

At $72 \mathrm{~h}$ after the addition of CCK-8, cell proliferation inhibition was lower in the HL-60-LS3+OA cells than in the HL-60+OA cells, with absorbance values of $2.16 \pm 0.27$ vs. $1.48 \pm 0.12(\mathrm{P}<0.01)$. However, there was no difference in proliferation between the HL-60-LS3+OA and HL-60 cells. These results indicated that the lentiviral-mediated gene silencing of cav-1 hampered OA-induced inhibition of HL-60 cell proliferation (Fig. 3B).

Detection of apoptosis. At $24 \mathrm{~h}$ after each treatment, the apoptosis levels of each group were detected using flow cytometry (Fig. 4). The apoptotic rates of the HL-60, HL-60+OA, HL-60-LC, HL-60-LC+OA and HL-60-LS3+OA groups were $5.79 \pm 2.30,14.78 \pm 5.39,22.8 \pm 4.64,41.19 \pm 5.07$ and $6.55 \pm 2.23 \%$, respectively. The results showed that both OA treatment $(\mathrm{P}<0.05)$ and cav-1 overexpression $(\mathrm{P}<0.01)$ promoted HL-60 cell apoptosis, and the apoptosis levels of the HL-60-LC+OA cells were significantly higher than that of the HL-60+OA $(\mathrm{P}<0.01)$ or HL-60-LC cells $(\mathrm{P}<0.01)$. These data suggest that $\mathrm{OA}$ and $\mathrm{CAV}-1$ promote apoptosis of HL-60 cells, and the joint treatment had a more significant pro-apoptotic effect (Fig. 4A).

The rate of apoptosis of the HL-60-LS3+OA cells did not significantly differ from that of the HL-60 cells; however, the rate of $8.23 \pm 3.16 \%(\mathrm{P}<0.05)$ was lower than that of the HL-60+OA cells, indicating that silencing of the cav-1 gene hindered OA promotion of HL-60 cell apoptosis (Fig. 4B).

Detection of cell cycle progression. We performed cell cycle analyses to further examine the effects of OA administration, cav-1 overexpression and cav-1-siRNA on the HL-60 cell cycle (Fig. 5). The percentage of HL-60+OA 
A

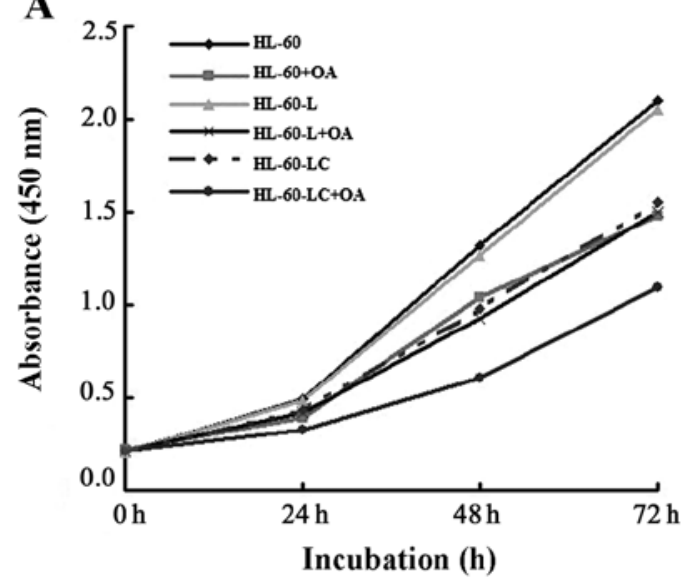

B

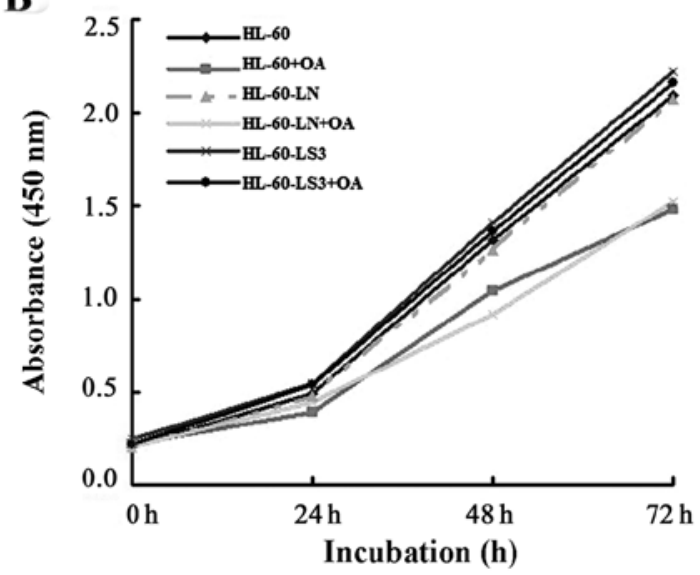

Figure 3. Cell proliferation of each group was detected using CCK-8 assay. (A) The effects of CAV-1 and OA on HL-60 cell proliferation. (B) The effects of OA on HL-60 cell proliferation after cav-1 silencing. ${ }^{*} \mathrm{P}<0.05,{ }^{* *} \mathrm{P}<0.01$, samples compared with HL-60 cells. ${ }^{\# \#} \mathrm{P}<0.01$, samples compared with HL-60+OA cells. ${ }^{\triangle} \triangle \mathrm{P}<0.01$, samples compared with HL-60-LC cells.
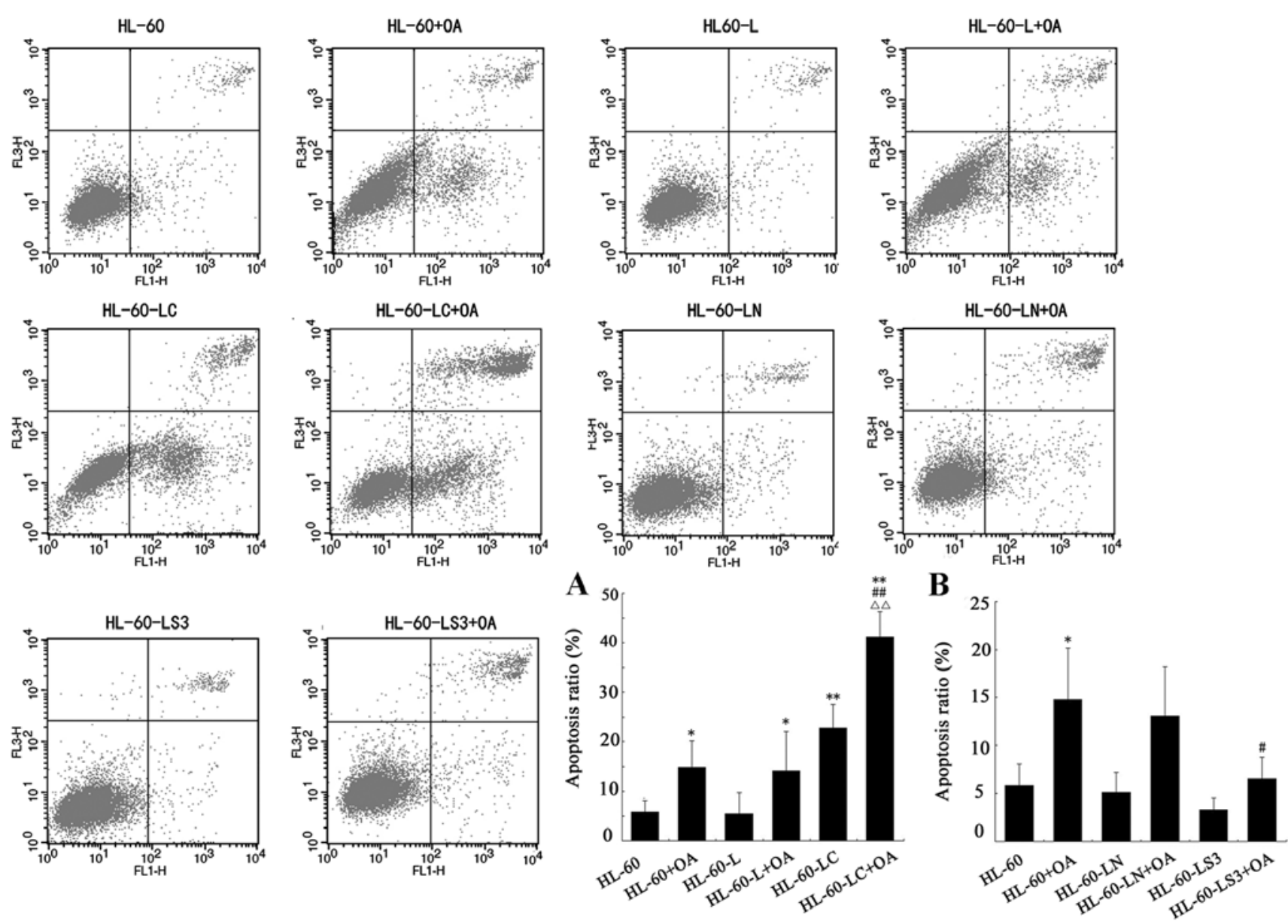

Figure 4. The apoptotic ratio of each group of cells as detected by flow cytometry. (A) The effects of cav-1 and OA on HL-60 cell apoptosis. (B) The effects of OA on HL-60 cell apoptosis after cav-1 silencing. ${ }^{*} \mathrm{P}<0.05,{ }^{* *} \mathrm{P}<0.01$, samples compared with HL-60 cells. ${ }^{\# \#} \mathrm{P}<0.01$, samples compared with HL-60+OA cells. $\triangle \triangle \mathrm{P}<0.01$, samples compared with HL-60-LC cells.

cells and HL-60-LC cells in the G1 phase increased by $7.0 \pm 1.1(\mathrm{P}<0.05)$ and $10.9 \pm 1.9 \%(\mathrm{P}<0.01)$, respectively. The percentage of HL-60-LC+OA cells in the G1 phase increased by $12.3 \pm 0.6(\mathrm{P}<0.01)$ and $8.4 \pm 1.4 \%(\mathrm{P}<0.01)$ compared with the HL-60+OA and HL-60-LC cells, respectively. Both OA and CAV-1 arrested the HL-60 cells at the G1 phase of the cell cycle, and the cell cycle arrest was more significant in the CAV-1+OA cells (Fig. 5A).

The percentage of HL-60-LS3+OA cells in the G1 phase did not differ from that of the HL-60 group but was decreased 

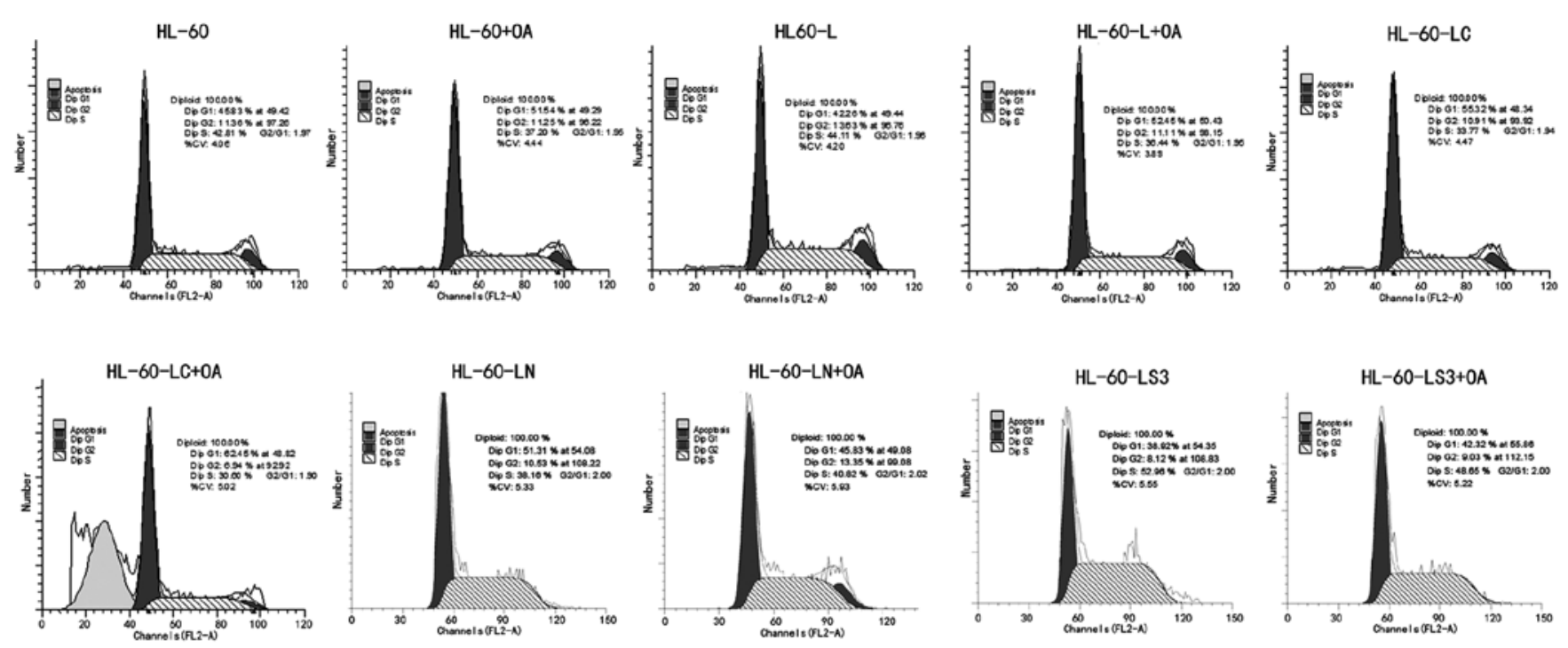

A

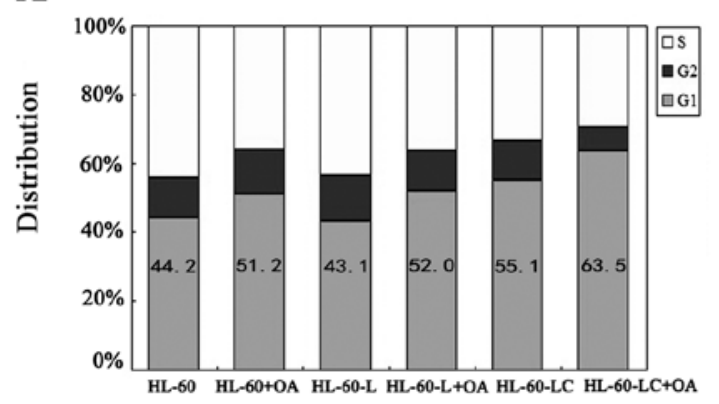

B

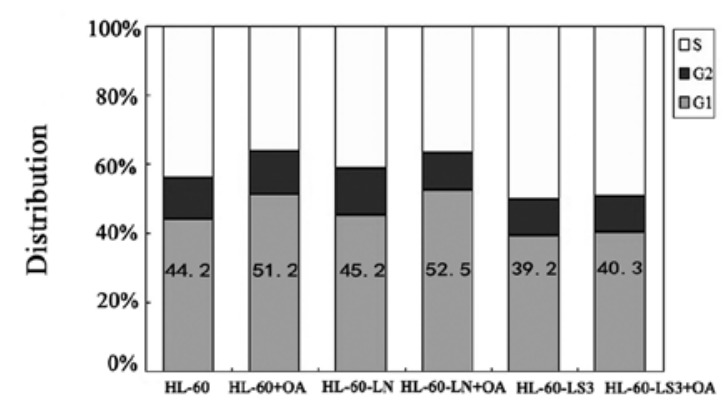

Figure 5. The cell cycle distribution of each group of cells as detected by flow cytometry. (A) The effects of cav-1 and OA on the HL-60 cell cycle. (B) The effects of OA on the HL-60 cell cycle after cav-1 silencing.

by $10.9 \pm 3.4 \%(\mathrm{P}<0.01)$ compared with that of the HL-60+OA group, indicating that cav-1 gene silencing hampered the $\mathrm{OA}$ effect on the G1 arrest of HL-60 cells (Fig. 5B).

Western blot analysis results. The PI3K/AKT/mTOR signaling pathway is a key pathway of tumorigenesis and tumor development. Therefore, we detected not only CAV-1 protein levels and its phosphorylation but also the levels of P110 $\alpha$, AKT, p-AKT (Ser473), p-AKT (Thr308) and mTOR to determine the relationships between OA, CAV-1 and the PI3K/AKT/ mTOR signaling pathways.

Lentivirus-mediated overexpression of cav-1 and $O A$ treatment increased the expression of $C A V-1$ and $C A V-1$ (Tyr14) phosphorylation levels and cav-1 silencing inhibited the OA effect on CAV-1 phosphorylation. Both OA and Lv-CAV-1 upregulated CAV-1 expression levels, increased CAV-1 $\alpha$ phosphorylation at Tyr14 and decreased AKT phosphorylation at Ser473 and Thr308. The decreased CAV-1 expression in HL-60-LS3 cells indicated the effect of cav-1 silencing. CAV-1 expression in HL-60-LS3+OA cells was significantly lower than in HL-60+OA cells $(\mathrm{P}<0.01)$ and showed no differences compared with HL-60 cells, indicating that cav-1 siRNA blocked OA-induced overexpression of CAV-1 (Fig. 6).

Cav-1 overexpression and OA treatment suppressed the PI3K/AKT/mTOR signaling pathway; OA did not suppress the pathway after cav-1 silencing. The expression levels of $\mathrm{P} 110 \alpha$, p-AKT (Ser473), p-AKT (Thr308) and mTOR were decreased in the HL-60+OA, HL-60-LC and HL-60-LC+OA cells. The smallest decrease was observed in the HL-60-LC+OA cells. P110 $\alpha$ expression levels were increased in the HL-60-LS3 cells $(\mathrm{P}<0.05)$. The levels of P110 $\alpha$, p-AKT (Ser473), p-AKT (Thr308) and mTOR were all lower in the HL-60+OA cells compared with the HL-60-LS3+OA cells. Compared with the HL-60 cells, the levels of P110 $\alpha$, p-AKT (Ser473), p-AKT (Thr308) and mTOR did not change in the HL-60-LS3+OA cells (Fig. 6). These results indicated that silencing cav-1 upregulated $\mathrm{P} 110 \alpha$ expression and blocked the inhibitory effect of OA on the PI3K/AKT/mTOR signaling pathway. It is likely that OA regulated cav-1 expression to alter the downstream $\mathrm{PI} 3 \mathrm{~K} / \mathrm{AKT} / \mathrm{mTOR}$ signaling pathways, and CAV-1 is likely a key factor in this regulatory cascade.

\section{Discussion}

Chemotherapy and bone marrow transplantation have traditionally been the main treatments for leukemia. Hematopoietic stem cell transplantation is difficult to widely implement due to matching difficulties, while chemotherapy is toxic to the normal cells of the body, making many patients abandon treatment due to intolerance to side effects. Oleanolic acid is widely found in plants such as privet fruit and the mileensis 

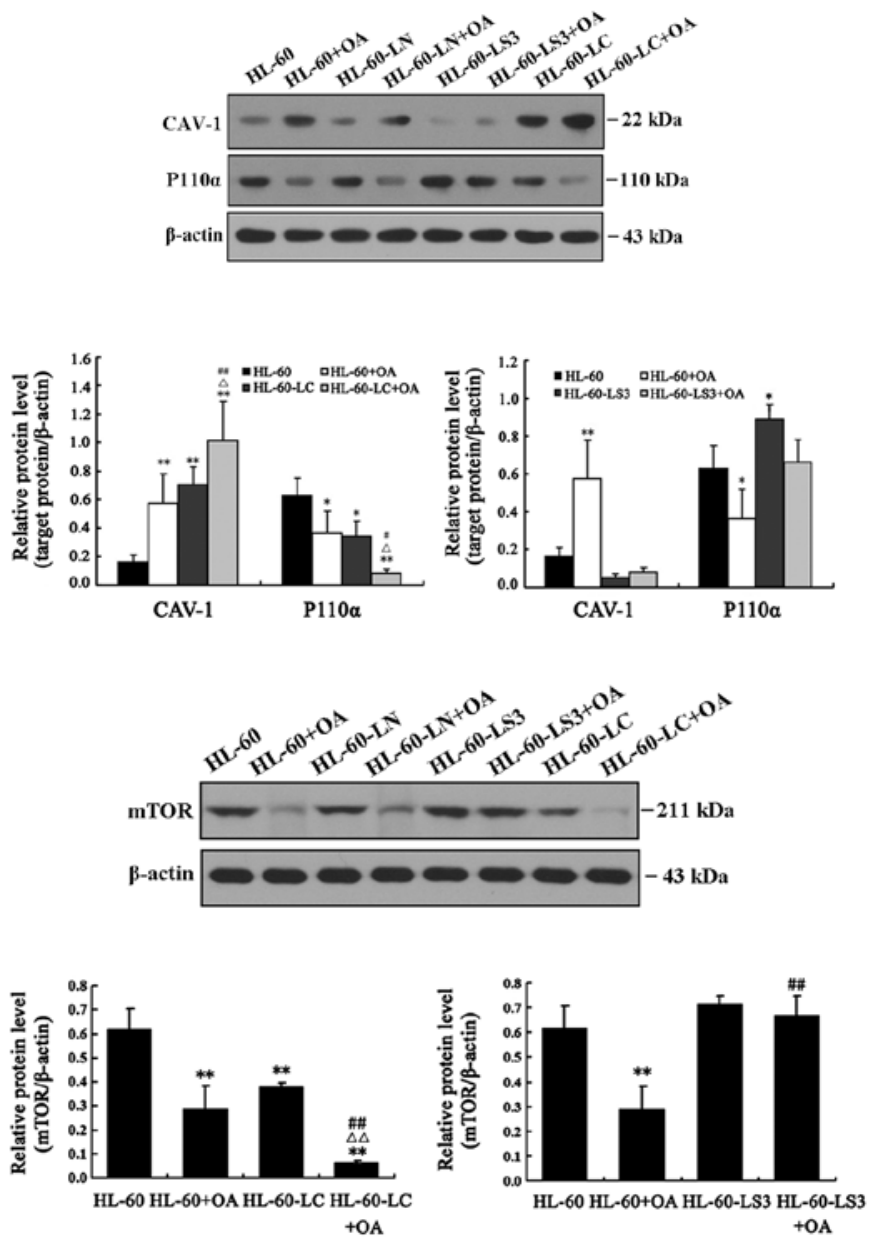
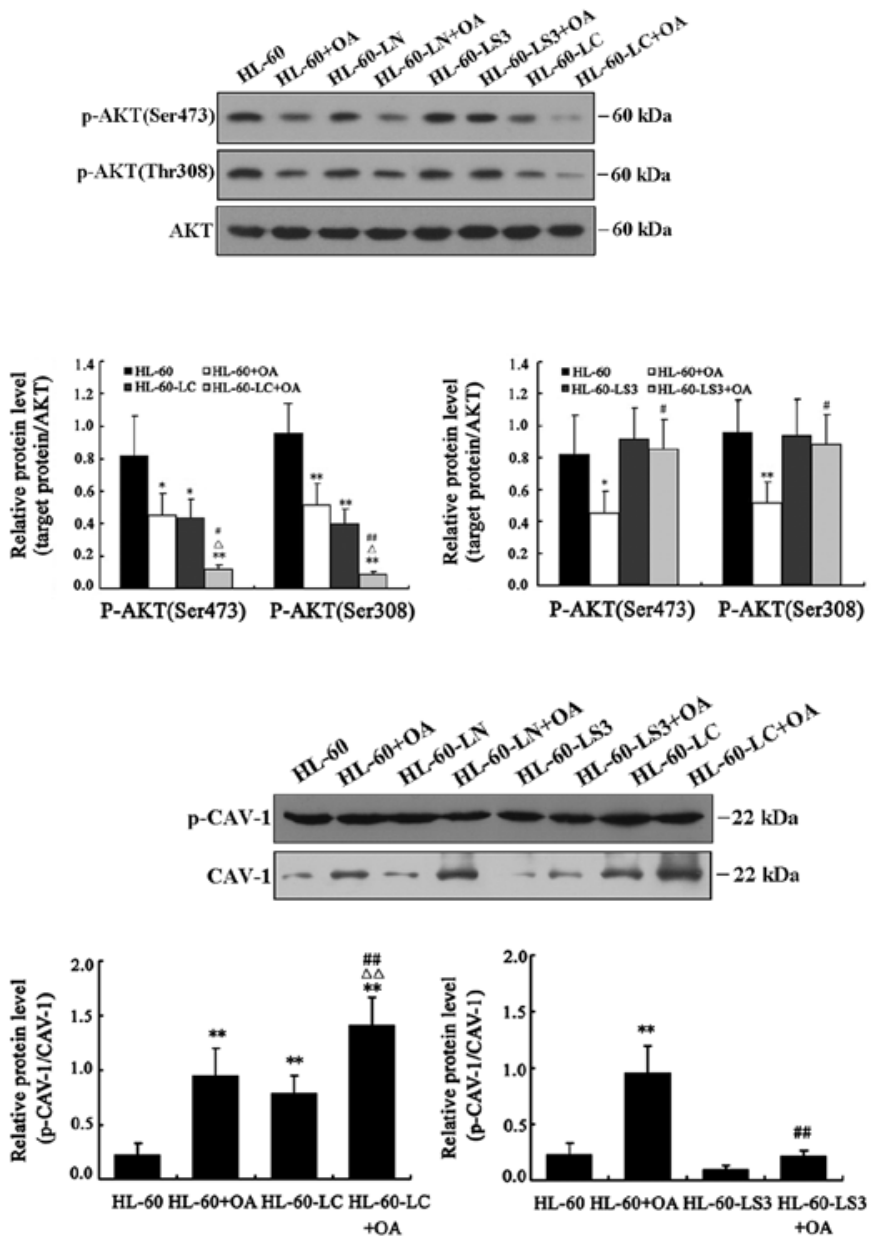

Figure 6. Expression of CAV-1, P110 $\alpha$, p-AKT (Ser473), p-AKT (Thr308), mTOR and p-CAV-1 in each group of cells. * $<<0.05$, ${ }^{* *} \mathrm{P}<0.01$, samples compared with HL-60 cells. ${ }^{\# \prime} \mathrm{P}<0.01$, samples compared with HL-60+OA cells. ${ }^{\triangle} \triangle \mathrm{P}<0.01$, samples compared with HL-60-LC cells.

whole plant. It was found in 1992 that as an active ingredient in the methanol extract of clove, OA induced differentiation of murine leukemia cells and inhibited HL-60 cell proliferation (22). In vitro experiments also showed that OA inhibited tumor growth and reduced radiation-induced damage to hematopoietic tissues (23). OA has been unexpectedly linked to CAV-1 and the PI3K pathway, and this study was designed to further explore the mechanism of $\mathrm{OA}$ in leukemia and to determine whether CAV-1 plays a key role.

The cav-1 gene plays a role as a tumor suppressor in HL-60 cells. cav-1 plays an important role in tumorigenesis and the development of leukemia. To study the differences in cav-1 gene expression between leukemia tumor cells and normal human leukocytes, we chose the ALL cell line SUP-B15, the ANLL cell lines HL-60 and THP-1, the CML cell line K562 and CLL primary tumor cells (named CLP) as the experimental subjects and found low cav-1 mRNA and protein expression in all cell lines. The HL-60 cell line with the lowest cav-1 expression was selected for lentivirus-mediated cav-1 gene transfection. The results showed that overexpression of cav-1 inhibited HL-60 cell proliferation, promoted apoptosis, provoked cell cycle arrest in the G1 phase and suppressed PI3K/AKT/mTOR pathway activity. Thus, cav-1 plays a role as a tumor suppressor in HL-60 cells.
The CAV-1 and PI3K/AKT/mTOR pathways are involved in HL-60 cell proliferation inhibition, promotion of apoptosis and cell cycle arrest in the G1 phase by OA. The nature of carcinogenesis is the deregulation of signal transduction pathways leading to unlimited cell proliferation. Searching for targets has been a focus of anticancer drug discovery in recent years. The study of CAV-1 and PI3K/AKT/mTOR signaling pathways has recently attracted attention and has been mainly limited to mouse embryonic stem cells. Estradiol $17 \beta$ stimulates the proliferation of mouse ES cells partly by upregulating CAV-1 through the Src, PI3K and ERK1/2 pathways (24); the epidermal growth factor can induce DNA synthesis and cell migration, in which CAV-1 plays a potential role through the Src, FAK and PI3K/AKT signaling pathways (25). Silencing the cav-1 gene can also activate the estrogen receptor 36-dependent PI3K/AKT pathway (26). CAV-1 participates in fibronectin's induction of mouse embryonic stem cells through the FAK, RhoA, PI3K/AKT and ERK1/2 pathways (27). The relationship between CAV-1 and PI3K/AKT/mTOR in leukemia has not yet been reported.

This experiment verified that OA not only upregulated CAV-1 expression but also enhanced CAV-1 phosphorylation. At the same time, OA treatment inhibited the PI3K/AKT pathway, leading to a series of changes in the activities of $\mathrm{P} 110 \alpha, \mathrm{AKT}$ in the pathway and downstream mTOR. The 
cav-1 overexpression in HL-60 cells via transfection caused changes in cell proliferation, apoptosis, cell cycle and the PI3K pathway, consistent with OA treatment. These results suggest that OA likely increases cav-1 expression by increasing its transcription or translation efficiency or acting on its promoter, thereby inhibiting the PI3K/AKT/mTOR signaling pathway to trigger a series of biological reactions.

OA improves CAV-1 protein phosphorylation in $H L-60$ cells. Using real-time quantitative RT-PCR, Tsuji et al demonstrated that in adult T-cell leukemia cell lines, $\mathrm{CpG}$ site methylation of the caveolin promoter was irrelevant to caveolin expression levels, and that other mechanisms may be involved in the regulation of caveolin expression (28). The changes of cav-1 epigenetic modification in AML will be the focus of future studies. We considered the change in CAV-1 phosphorylation while detecting the CAV-1 protein expression level. CAV-1 $\alpha$ has 31 more amino acid residues than CAV-1 $\beta$. It is widely believed that CAV-1 $\alpha$ phosphorylation at Tyr14, which is lacking in the $\beta$ isoform, is the key for tumorigenesis and development; this phosphorylation site could be the key factor causing the two isoforms to function differently. Therefore, in the present study, we detected p-CAV-1 (Tyr14) of the $\alpha$ isoform, and the results confirmed that OA increased not only CAV-1 protein expression levels but also its phosphorylation at Tyr14. p-CAV-1 may be the active form of CAV-1 that regulates cell proliferation, apoptosis and cell cycle progression by regulating the PI3K/AKT pathway.

CAV-1 increases HL-60 cell sensitivity to OA. After OA treatment in cav-1-overexpressing cell lines, HL-60 cell proliferation activity decreased, the apoptotic rate increased, the percentage of cells in the G1 phase increased, and the $\mathrm{PI} 3 \mathrm{~K} / \mathrm{AKT} / \mathrm{mTOR}$ pathway was significantly inhibited. The combination of CAV-1 and OA was significantly more effective than any single factor, suggesting that CAV-1 can improve HL-60 cell sensitivity to OA. Gene therapy supplemented by clinical medicine will be a novel treatment for AML drug resistance.

$C A V-1$ is a key factor in OA function. After cav-1 gene silencing by RNA interference, OA treatment could no longer regulate HL-60 cell proliferation, apoptosis, cell cycle and the PI3K pathway, i.e., CAV-1 shRNA hindered OA functioning in HL-60 cells. CAV-1 plays a very important role as a key factor for OA function in HL-60 cells.

Cav-1 acts as an oncogene according to some studies. For instance, the CAV-1 expression level is increased in renal cancer cells, and the survival rate in patients with CAV-1 is significantly lower than that of CAV-1-negative patients (29). CAV-1 showed an effect on anoikis resistance in non-small cell lung cancer, and CAV-1-positive patients have a poor prognosis (30). There have also been studies suggesting that cav-1 acts as a tumor-suppressor gene. For example, mutations at codon 132 and 141 of the cav-1 gene have been found in breast cancer (31). CAV-1 can play a regulatory role in tumors through a variety of pathways, such as PI3K/AKT (32), MEK/ERK, RAS and MAPK. Cav-1 gene deletion mutation, a high density of methylation at $\mathrm{CpG}$ loci in the promoter region (33), abnormal Y14 phosphorylation of the CAV-1 $\alpha$ isoform (34), abnormal activation or upregulated expression of CAV-1 by upstream transcription factors and signaling proteins can cause cancer. The results of this experiment found lower cav-1 expression levels in AML tumor cells than in normal white blood cells, suggesting cav-1 to be a possible tumor-suppressor gene. OA can block cell cycle progression through ERK-P53, promote apoptosis through the mitochondrial pathway, inhibit the mTOR signaling pathway (7) or downregulate $\mathrm{Bcl}-2$ to upregulate $\mathrm{Bax}$ and $\mathrm{Bad}$ to achieve antitumor effects (8); VEGF is also involved in the OA inhibitory effects on proliferation, invasion and tube formation by endothelial cells (10). The present study suggests that the cav-1 gene is a new target for the treatment of AML through cav-1 overexpression in HL-60 cells and provides a potential new strategy for research and development of new drugs for AML treatment and drug resistance. As a natural medicinal extract, OA can specifically increase CAV-1 protein expression levels in this target and inhibit the PI3K/AKT pathway. OA effectiveness was lost after cav-1 silencing, indicating that CAV-1 plays a key role in how OA affects HL-60 cells suggesting that OA can be used in AML therapy targeting CAV-1. However, how OA promotes $\mathrm{CAV}-1$ expression, whether $\mathrm{CAV}-1$ protein directly or indirectly inhibits the PI3K pathway, and where the anchor point is located require further research.

\section{Acknowledgements}

This study was supported by the National Natural Science Foundation of China (no. 81070428), the Natural Science Foundation of Heilongjiang Province (D201024), the Heilongjiang Provincial Health Department Scientific Research Project (no. 2010-484), the Heilongjiang Provincial Health Department Scientific Research Project (no. 2013-176), the Jiamusi University Key Scientific Research Project of Heilongjiang Provincial Health Department Scientific Research Projects (no. 2010-228), Heilongjiang Province Postdoctoral Scientific Research Startup Funding (no. LBH-Q12005) and Mudanjiang City, Heilongjiang Province Science and Technology Program (no. Z2013s048).

\section{References}

1. Huang WL: Signal Transduction. Beijing People's Medical Publishing House Press, Beijing, pp151-199, 2005.

2. Zhou L, Ding Y, Chen W, Zhang P, Chen Y and Lv X: The in vitro study of ursolic acid and oleanolic acid inhibiting cariogenic microorganisms as well as biofilm. Oral Dis 19: 494-500, 2013.

3. Wang J, Shan A, Liu T, Zhang C and Zhang Z: In vitro immunomodulatory effects of an oleanolic acid-enriched extract of Ligustrum lucidum fruit (Ligustrum lucidum supercritical $\mathrm{CO}_{2}$ extract) on piglet immunocytes. Int Immunopharmacol 4: 758-763, 2012.

4. Mapanga RF, Rajamani U, Dlamini N, et al: Oleanolic acid: a novel cardioprotective agent that blunts hyperglycemia-induced contractile dysfunction. Plos One 7: e47322, 2012.

5. Zhang H, Zhang Y, Jiang YP, et al: Curative effects of oleanolic acid on formed hypertrophic scars in the rabbit ear model. Evid Based Complement Alternat Med 2012: 837581, 2012.

6. Wang X, Bai H,Zhang X, et al: ERK-p53-mediated cell cycle arrest and mitochondrial-dependent apoptosis. Carcinogenesis 34 : 1323-1330, 2013.

7. Zhou R, Zhang Z, Zhao L, et al: Inhibition of mTOR signaling by oleanolic acid contributes to its anti-tumor activity in osteosarcoma cells. J Orthop Res 29: 846-852, 2011. 
8. Pratheeshkumar P and Kuttan G: Oleanolic acid induces apoptosis by modulating p53, Bax, Bcl-2 and caspase-3 gene expression and regulates the activation of transcription factors and cytokine profile in B16F. J Environ Pathol Toxicol Oncol 30: 21-31, 2011

9. Feng L, Au-Yeung W, Xu YH, Wang SS, Zhu Q and Xiang P: Oleanolic acid from Prunella Vulgaris L. induces SPC-A-1 cell line apoptosis via regulation of $\mathrm{Bax}, \mathrm{Bad}$ and $\mathrm{Bcl}-2$ expression. Asian Pac J Cancer Prev 12: 403-408, 2011.

10. Wei JT, Liu M, Liu HZ, Zhao J, Xiao L, Han LJ and Lin XK Oleanolic acid inhibits proliferation of HUVECs, and inhibits migration and tube formation via VEGF pathway. Yao Xue Xue Bao 47: 1457-1462, 2012 (In Chinese).

11. Choi CY, You HJ and Jeong HG: Nitric oxide and tumor necrosis factor-alpha production by oleanolic acid via nuclear factor-kappaB activation in macrophages. Biochem Biophys Res Commun 288: 49-55, 2001.

12. Schwencke C, Braun-Dullaeus RC, Wunderlich C and Strasser RH: Caveolae and caveolin in transmembrane signaling: Implications for human disease. Cardiovascular Res 70: 42-49, 2006.

13. Yi JS, Mun DG, Lee H, et al: PTRF/cavin-1 is essential for multidrug resistance in cancer cells. J Proteome Res 12: 605-614, 2013.

14. Lavie Y, Fiucci G and Liscovitch M: Up-regulation of caveolae and caveolar constituents in multidrug-resistant cancer cells. J Biol Chem 273: 32380-32383, 1998.

15. Fang K, Fu W, Beardsley AR, Sun X, Lisanti MP and Liu J: Overexpression of caveolin-1 inhibits endothelial cell proliferation by arresting the cell cycle at G0/G1 phase. Cell Cycle 6 : 199-204, 2007.

16. Zou H, Stoppani E, Volonte D and Galbiati F: Caveolin-1, cellular senescence and age-related diseases. Mech Ageing Dev 132: 533-542, 2011.

17. Bai L, Deng X, Li J, et al: Regulation of cellular senescence by the essential caveolar component PTRF/Cavin-1. Cell Res 21: 1088-1101, 2011.

18. Martinez-Outschoorn UE, Whitaker-Menezes D, Lin Z, et al: Cytokine production and inflammation drive autophagy in the tumor microenvironment: role of stromal caveolin-1 as a key regulator. Cell Cycle 10: 1784-1793, 2011.

19. Liu Y, Wang Y, Shi D and Zou W: Autophagy and Caveolin-1 in cancer: a review. Chen Wu Gong Chen Xue Bao 28: 912-917, 2012 (In Chinese)

20. Pang A, Au WY and Kwong YL: Caveolin-1 gene is coordinately regulated with the multidrug resistance 1 gene in normal and leukemic bone marrow. Leuk Res 28: 973-977, 2004.

21. Sawada S, Ishikawa C, Tanji $\mathrm{H}$, et al: Overexpression of caveolin-1 in adult T-cell leukemia. Blood 115: 2220-2230, 2010.
22. Umehara K, Takagi R, Kuroyanagi M, Ueno A, Taki T and Chen YJ: Studies on differentiation-inducing activities of triterpenes. Chem Pharm Bull (Tokyo) 40: 401-405, 1992.

23. Hsu HY, Yang JJ and Lin CC: Effects of oleanolic acid and ursolic acid on inhibiting tumor growth and enhancing the recovery of hematopoietic system postirradiation in mice. Cancer Lett 111: 7-13, 1997.

24. Park JH, Lee MY and Han HJ: A potential role for CAV1 in estradiol-17 beta-induced proliferation of mouse embryonic stem cells: involvement of Src, PI3K/Akt, and MAPKs pathways. Int J Biochem Cell Biol 41: 659-665, 2009.

25. Park JH and Han HJ: CAV1 plays important role in EGF-induced migration and proliferation of mouse embryonic stem cells: involvement of PI3K/Akt and ERK. Am J Physiol Cell Physiol 297: C935-C944, 2009.

26. Feng S, Wang Y, Wang X, et al: CAV1 gene silencing promotes the activation of PI3K/AKT dependent on ERalpha36 and the transformation of MCF10ACE. Sci China Life Sci 53: 598-605, 2010.

27. Park JH, Ryu JM and Han HJ: Involvement of CAV1 in fibronectin-induced mouse embryonic stem cell proliferation: role of FAK, RhoA, PI3K/Akt, and ERK 1/2 pathways. J Cell Physiol 226: 267-275, 2011.

28. Tsuji Y, Nakagawa T, Hatanaka M, Takeuchi T, Matsumoto E, Takenaka $\mathrm{H}$ and Shimizu A: Quantification of caveolin isoforms using quantitative real-time RT-PCR, and analysis of promoter $\mathrm{CpG}$ methylation of caveolin-1alpha in human T cell leukemia cell lines. Int J Mol Med 18: 489-495, 2006

29. Joo HJ, Oh DK, Kim YS, Lee KB and Kim SJ: Increased expression of CAV1 and microvessel density correlates with metastasis and poor progrosis in clear cell renal cell carcinoma. BJU Int 93: 291-296, 2004.

30. Chunhacha P and Chanvorachote P: Roles of Caveolin-1 on anoikis resistance in non small cell lung cancer. Int J Physiol Pathophysiol Pharmacol 4: 149-155, 2012.

31. Lee MY, Lee SH, Park JH and Han HJ: Interaction of galectin-1 with caveolae induces mouse embryonic stem cell proliferation through the Src, ERas, Akt and mTOR signaling pathways. Cell Mol Life Sci 66: 1467-1478, 2009.

32. Ryu JM and Han HJ: L-threonine regulates G1/S phase transition of mouse embryonic stem cells via PI3K/Akt, MAPKs, and mTORC pathways. J Biol Chem 286: 23667-23678, 2011.

33. Rao X, Evans J, Chae H, et al: CpG island shore methylation regulates caveolin-1 expression in breast cancer. Oncogene 32: 4519-4528, 2013.

34. Felicetti F, Parolini I, Bottero L, et al: Caveolin-1 tumorpromoting role in human melanoma. Int J Cancer 125: 1514-1522, 2009. 